\title{
Erratum to: Receipt of Transition Services Within a Medical Home: Do Racial and Geographic Disparities Exist?
}

\author{
Nicole Richmond • Tri Tran · Susan Berry
}

Published online: 11 February 2011

(C) Springer Science+Business Media, LLC 2011

\section{Erratum to: Matern Child Health J (2010) \\ DOI: $10.1007 / \mathrm{s} 10995-010-0635-2$}

Table 7 has been revised to correct the placement error for the household Federal Poverty Level (FPL) variable reference. In the corrected table, the reference household income level is equal to or below 200\% FPL. The odds ratios published in the original article are correct, and thus remain the same in the amended table. The edit made in this table does not change either the results or the discussion sections.

The online version of the original article can be found under doi:10.1007/s10995-010-0635-2.

N. Richmond $(\bowtie) \cdot$ S. Berry

Louisiana State University Health Sciences Center, School of

Medicine, Department of Pediatrics/Louisiana Office of Public

Health, Children's Special Health Services Program, 1010

Common Street Suite 610, New Orleans, LA 70112, USA

e-mail: Nicole.Richmond@la.gov

S. Berry

e-mail: Susan.Berry@la.gov

T. Tran

Louisiana State University Health Sciences Center, School of Medicine, Department of Pediatrics/Louisiana Office of Public Health, Maternal and Child Health Program, 1010 Common Street Suite 2710, New Orleans, LA 70112, USA

e-mail: Tri.Tran@la.gov
Table 7 Racial disparity for receipt of healthcare transition among YSHCN from the Southern Region, 2005-2006 National Survey of CSHCN

\begin{tabular}{lll}
\hline Effects & $\begin{array}{l}\text { Unadjusted OR } \\
(95 \% \mathrm{CI})\end{array}$ & $\begin{array}{l}\text { Adjusted OR } \\
(95 \% \mathrm{CI}) *\end{array}$ \\
\hline Race/ethnicity & & \\
Non-hispanic white & $3.45(2.49-4.76)$ & $2.59(1.73-3.86)$ \\
Non-hispanic black & 1.00 & 1.00
\end{tabular}

Amount of time in past year condition affected child

$\begin{array}{lcl}\text { Never } & 5.37(3.53-8.16) & 3.94(2.53-6.15) \\ \text { Sometimes } & 2.28(1.50-3.47) & 2.13(1.36-3.35) \\ \text { Usually } & 1.09(0.62-1.90) & 1.03(0.55-1.91) \\ \text { Always } & 1.00 & 1.00 \\ \text { Youth has adequate insurance } & \\ \text { Yes } & 3.03(2.37-3.88) & 2.52(1.91-3.32) \\ \text { No } & 1.00 & 1.00 \\ \text { Household highest education level } & \\ <\text { High school } & 1.00 & 1.00 \\ \text { High school } & 3.37(1.99-5.70) & 2.56(1.33-4.89) \\ >\text { High school } & 6.82(4.25-10.94) & 3.02(1.65-5.54) \\ \text { Household federal poverty level } & \\ \leq 200 \% \text { FPL } & 1.00 & 1.00 \\ >200 \% \text { FPL } & 3.44(2.62-4.50) & 1.82(1.31-2.53)\end{array}$

* Final Model included 2,666 observations (89.5\% of Southern region study sample) 